\title{
Prevalence of Helicobacter Pylori among different groups of Nephrotic children
}

\author{
Author running head : Affifi M and Sharaf M \\ Correspondence :Maged El-Sayed Affifi ,MD 11 Mahmoud Ghoneim Street, Nasr City , \\ Cairo,Egypt
}

E-mail: maged_elsayed@yahoo.com

Keywords : H.Pylori ,N.S and stool antigen test

Abbreviations: AGA: American Gastroenterology, GI:Gastrointestinal , H.Pylori :

Helicobacter Pylori, N.S :Nephrotic syndrome, SAT: Stool Antigen Test , SDNS: Steroid Dependent Nephrotic Syndrome, SSNS: Steroid sensitive nephrotic syndrome , SRNS : Steroid Resistant Nephrotic Syndrome

Original article: Prevalence of Helicobacter Pylori among different groups of Nephrotic children

By

Maged El-Sayed Affifi (Fellow of Pediatrics-children's Hospital-Ain Shams University )

And Mohamed Abdel-Moneim Sharaf (Lecturer of Pediatrics-Ain Shams University)

\begin{abstract}
Affifi M and Sharaf M-Ain Shams University

Introduction : Gastritis and peptic ulcer are common in children with nephrotic syndrome (NS). This is because of immunospressive medications like steroids with proteinuria and subsequent hypoga mmeglobulinemia
\end{abstract}

Aim of the work: Is to study the prevalence of H.pylori infection among different groups of children suffering from NS

Subject and methods: This study was cross sectional-case controlled study conducted at pediatric nephrology clinic - Ain Shams University

It was done between July 2019 till November 2020. It included 86 children with different types of N.S and 36 healthy age and sex matched children as control group. All N.S children had stool analysis for parasites and H.pylori antigen with renel functions. N.S children were divided into three groups according to the response to steroids

Results :H.pylori infection is common in children with nephrotic than controls with a statistical signification difference $(p<0.05)$ H.pylori infection is statistically signification correlated with GI symptoms as vomity, and infection with parasites besides duration of NS infection

Conclusion: Stool antigen test is reliable test for detection of H.pylori infection .H.pylori infection is common in children with NS and related to GI complaints and parasitic infestations

Keywords : H.Pylori,N.S and stool antigen test 


\section{Introduction :}

Gastritis and pepticulcer disease are common in children with hypogammaglobulenemia as children with nephrotic syndrome ( NS ) ( 1 )

The diagnostic tests for for H.Pylori are 2 types :

Type 1 ( Invasive tests ) which include ( 2 )
A- PCR
B- Rapid urease test
C- Endoscopy with urease test

Stool antigen test ( SAT) is a non invasive diagnostic modules for H.Pylori infection. Both European and Japanese guidelines have indicated it for primary diagnosis.

SAT has high accuracy than serological test. The American Gastroenterology Association ( AGA ) recommended SAT for the diagnosis of H.Pylori infection. ( 4 )

\section{Aim of the work:}

Is to study the prevalence of H.Pylori infection among different groups of children suffering from NS.

\section{Subjects and Methods:}

Their ages ranged between $5 \mathrm{Y}-16 \mathrm{Y}$ with a mean age $(10.7+-5.3 Y)$

Children with NS were subdivided into further 3 groups according to the degree of steroids response:

Group 1: SSNS it included 32 patients

Group 2: SDNS it included 26 patients

Group 3: SRNS it included 28 patients

All the 3 groups (NS patients ) were evaluated by:
Type 2 ( Non invasive tests) which include
A- urea breath test
B- ELISA
C- H.Pylori antigen in stool.

Nephrotic children are at great risk of infection .

These children are suffering from severe proteinuria with subsequent hypogammaglobulenemia. Also these children are receiving steroids and other immunosupressive medications according to the type of their NS. ( 3 )

This study was cross sectional case controlled study.

This study was conducted at Pediatric Nephrology clinic, Children's Hospital, Ain Shams University.

Between July 2019 till November 2020

It included 86 children with NS and 36 healthy age and sex matched children as a control group.

Children with NS were 52 males ( $60.4 \%$ ) and 34 females

\section{$(39.6 \%)$}

1- Thorough history and clinical examination which included:

Gastrointestinal symptoms as ( Vomiting, dyspepsia, hematemesis , similar GI complain in family, poor appetite )

2- Stool analysis for parasites

3- H.Pylori antigen in stool

4- Duration of NS in months

5- Total duration of steroids treatment in months

6- S.Creatinine, blood urea and S.albumin

- Control group 36 children, were selected to the healthy, non relatives and non house hold contacts with our patients. They were 
subjected to the same thorough history and clinical examination of NS children.

- Inclusion criteria:

All patients with Idispathic NS with different categories of steroid responsiveness.

- Exclusion criteria :

1- Patients receiving antibutics, PPIS during the last 2 weeks

2- Patients below 4 years who may be non cooperative during history taking.

\section{Statistical Methods :}

Data were tabulated and subjected to analysis using Microsoft Excel version 5.0 and the Statistical Package for Social Science

Specificity of the test : is the ability of the test to exclude
Also , they had the test of detection of H.Pylori antigen in stool.

( SPSS ) version 11.0. The following methods were employed :

- Frequency distributions and percentage distributions.

- Mean, standard deviation and range of numerical data.

- Comparison of means using the Student ttest; testing differences between means for statistical significance.

- Non-numerical data were compared using the chi-square test.

- In general, P values less than 0.05 are considered significant, less than 0.01 highly significant and those below 0.001 very highly significant. ( 5 )

negatives :

True negatives

True negatives + false positives

Sensitivity of the test : is the ability of the test to exclude positives :

Sensitivity True positives

True positives + false negatives

Predictive test for negative results : percent of true negatives among all negatives :

Negative predictive $=\quad$ True negatives

True negatives + false negatives

Predictive test for positive results : percent of true positives among all positives :

Positive predictive $=\quad$ True positives

True positives + false positives 
Efficacy : Diagnostic power of the test :

Efficacy $=\quad$ true positives + true negatives

True positives + true negatives + false positive+false negatives ( 6 )

\section{Results :}

There was a statistical significance of H.Pylori infection between cases ( all group SSNS - SDNS SRNS ) and controls $\quad(\mathrm{P}<0.05)$ but there was no statistical significant difference among 3 groups.

There was a high statistical correlation of H.Pylori infection in relation to Hypertension, Excess Junk food intake and similar GI complain in family, documented H.Pylori case in family 0.001 )

Also , H.Pylori infection was statistically significant in relation to GI symptoms as vomity , hematemesis, dyspepsia and poor appetite $(\mathrm{P}<0.05)$

- H.Pylori infection was statistically significant in relation to infection with parasites as Giardia or E.histolytica $(\mathrm{P}<0.05)$

- H.Pylori infection was statistically significant in relation to duration of NS infection and total duration of steroids treatment $(\mathrm{P}<0.05)$ but non significant $(\mathrm{P}>0.05)$ in relation to the dose of steroids, the patients were receiving

- In addition, H.Pylori infection was statistical significant $(\mathrm{P}<0.05)$ in relation to low S.albumin

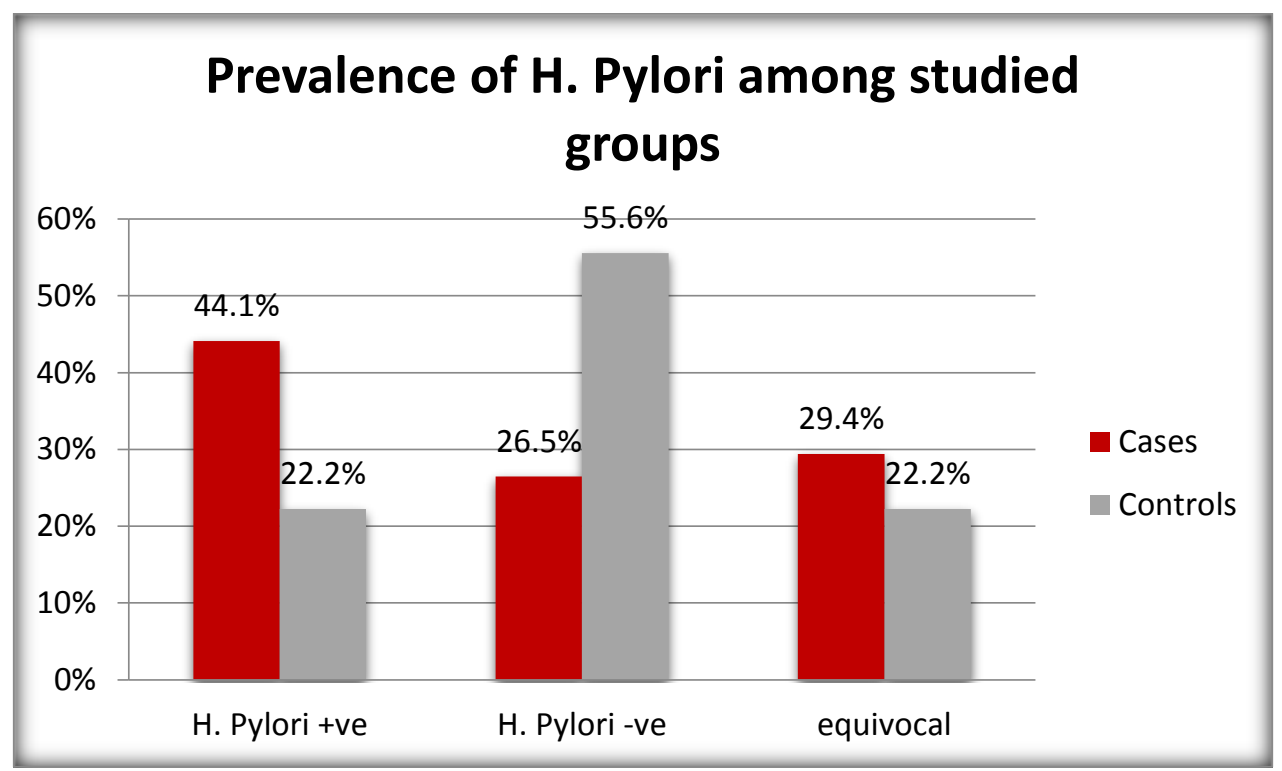



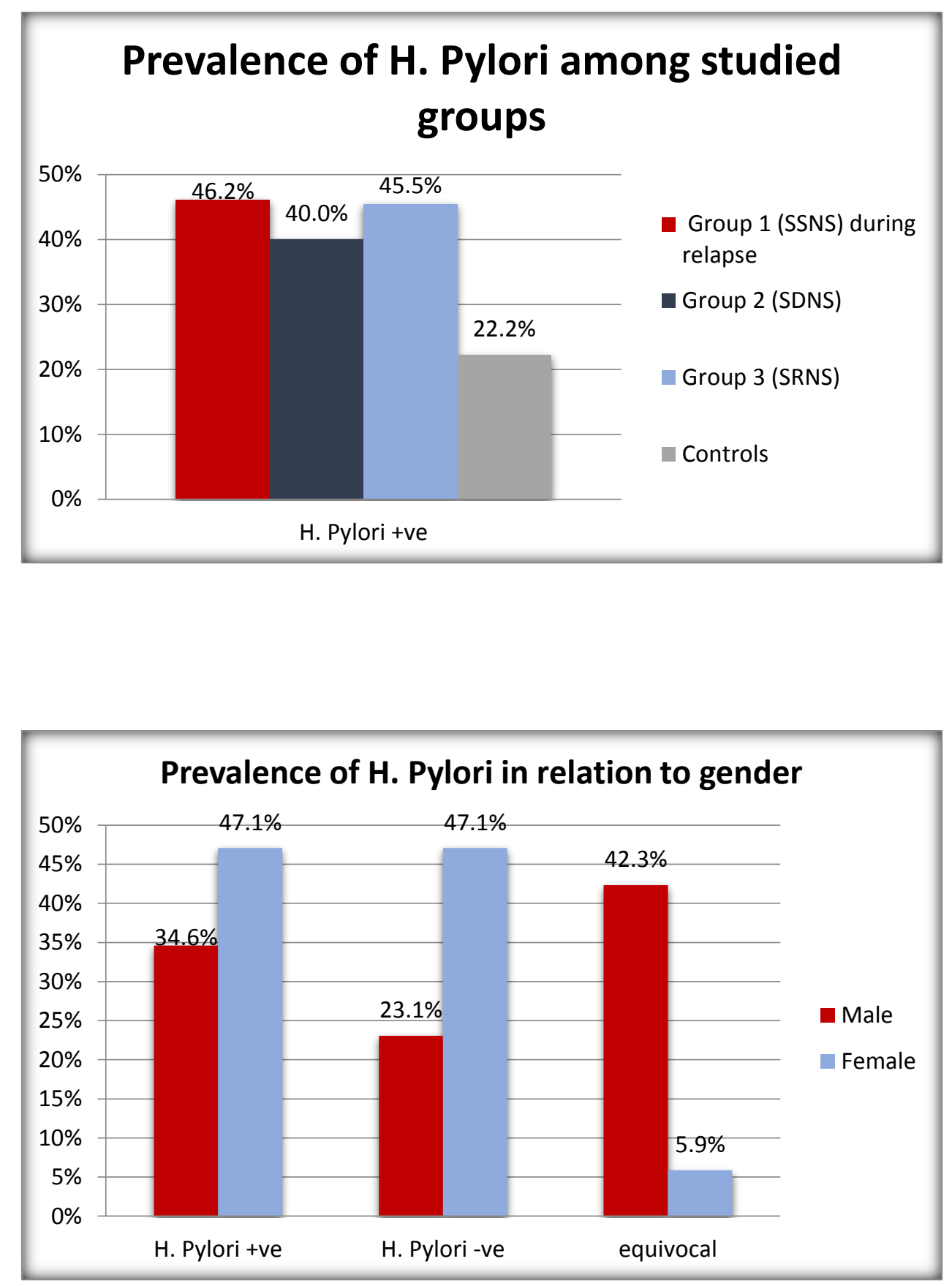

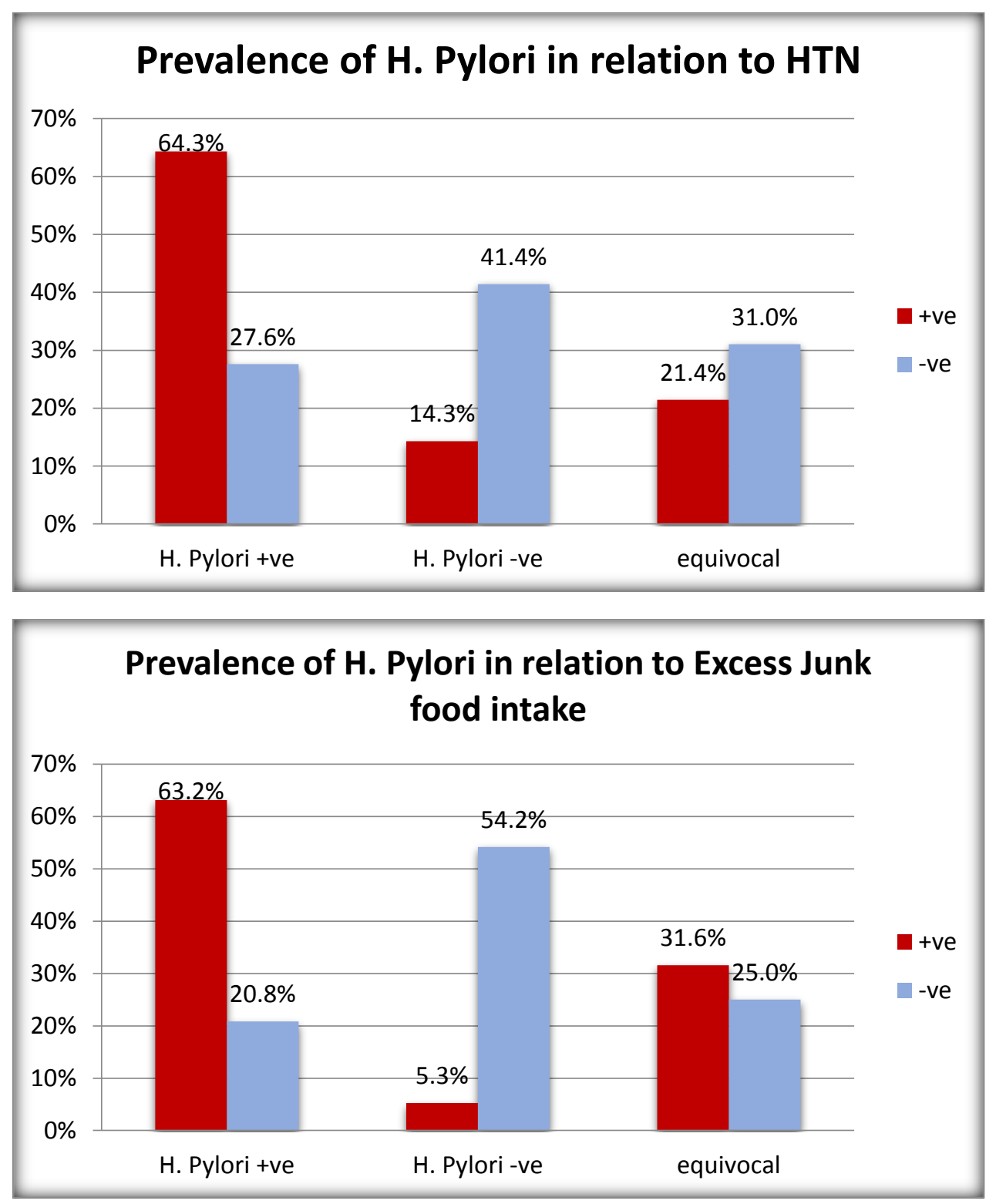

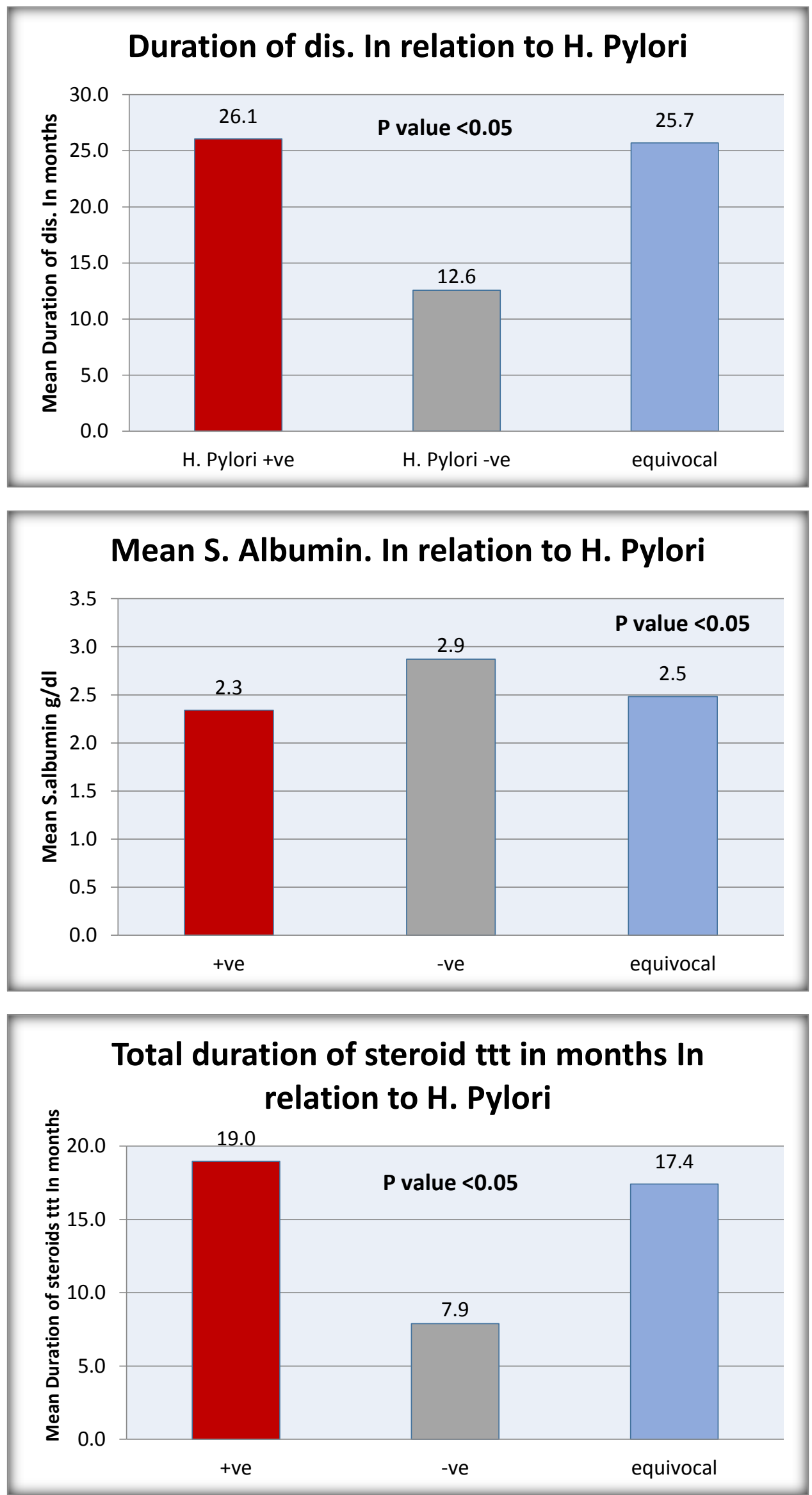


\section{Discussion :}

Since most of our nephrotic patients belong to low socioeconomic class and in addition steroids are the mean line of treatment in nephrotics, this was our stimulus for screening of H.Pylori in this group of patients. ( 7 )

We looked at 86 patients with nephrotic syndrome ( 52 males and 34 females ), for the prevalence of H.Pylori infection, they were randomly selected from the Pediatric Nephrology Clinic - Children's Hospital Ain Shams University with a ratio of males $>$ females. This gender ratio of selected patients coincides with the prevalence of NS among males more than females ( Farid et al., 1997 ) ( 8 )

Children included in the control group were selected to be non relatives, non household contacts to the patients. This concept fulfils the criteria stated by Miyaji et al., 2000 ( 9 ) that the acquisition of H.Pylori infection occurs by close contact with the infected individuals in early childhood especially via contact with infected mothers and other infected children.

In recent ESPGHAN ( European Society for Paediatric Gastroenterology , Hepatology and Nutrition ) and NASPGHAN ( North American Society for Paediatric Gastroenterology ,

( 10 ) Hepatology and Nutrition ) guidelines for H.Pylori infection in children, the recommendation is that the primary goal of clinical investigation of gastrointestinal symptoms is to determine the underlying cause of the symptoms and not solely the presence of H.Pylori infection.( 11 )

\section{Conclusion :}

1- Stool antigen test is a reliable, noninvasive test for detection of H.Pylori infection in children. It has a high index of specificity and sensitivity.
This coincides with our results which showed significant correlation $(\mathrm{P}<0.05$ ) with GI symptoms as vomiting, dyspepsia and poor appetite.

H.Pylori infection at our study had a high statistical correlation $(\mathrm{P}<0.001)$ in relation to hypertension and family history of GI complaint or documented H.Pylori infection at same family.

That is why our results in H.Pylori infection was statistical difference in children with nephrotic syndrome compared to control group $(\mathrm{P}<0.05)$. This was in contrast to Ibrahim et al.,2015 ( 12 ) who stated that there was no difference of H.Pylori infection between nephrotic children and controls. Because, this study did not mention how they selected their control group which were most probably relatives or neighbours of their nephrotic children.

H.Pylori infection had significant correlation ( $\mathrm{P}<$ 0.05 ) in relation to the duration of disease of NS and total duration of steroids treatment. This was matched with Sabbagh et al., 2015 ( 13 ) about diagnosis of H.Pylori infection among high risk children.

Stool antigen test has a high sensitivity, specificity, positive predictive value, negative predictive value and efficacy of $\quad(93.6 \%$, $100 \%, 100 \%, 87.3 \%$ and $96 \%$ respectively ) which coincides with the findings of Moubri et al., 2019. (14)

2- H.Pylori infection is common among children suffering from nephrotic syndrome than healthy one.

3- H.Pylori infection is related to GI complaints and family history of 
H.Pylori infection especially in immunocompromized children with NS.

\section{Recommendations :}

1- Screening of H.Pylori infection among children with NS. to be concerned with prolonged duration of treatment.

2- Eradication of parasitic Infestations in children with nephrotic syndrome as it is risk factor for H.Pylori infection.

3- Further studies to be done about SAT after treatment and eradication of H.Pylori infection in children.

\section{References}

1- RafeeyM and Nikvash S :"detection of Helicobacter pylori antigen in stool samples for diagnosis of infection in children (2007) Eastern Mediterranean health journal 13(5):1067,1072

2- Shimoyama T: " stool antigen tests for the menagement of Helicobacter pylori infection" (2013) :World J Gastroentral 19 (45) :8188-8191

3- KotileaK, KalachN, HomanM, BontemsP. Helicobacter pylori infection in pediatric patients: update on diagnosis and eradication strategies. Paediatr Drugs.2018;20(4):337-351.

4- ShimoyamaT, OyamaT, MatsuzakaM, DanjoK, NakajiS, FukudaS. Comparison of a stool antigen test and serology for the diagnosis of Helicobacter pylori infection in mass survey. Helicobacter. 2009; 14:8790

5- WilliamsF, (1981) : Relationship anelysis. In : Reasoning with statistics. 3rd edition. Holc,Rine Winston, New York. Pp.132.

6- KakiuchiT, OkudaM, HashiguchiK, ImamuraI, NakayamaA, MatsuoM.
Evaluation of a novel stool an antigen rapid test kit for the detection of Helicobacter pylori infection. J Clin Microbiol. 2019;57(3):e01825-18.

7- ChobotA, PorebsKaJ, KrzywickaA and etal (2019) “Association between Helicobacter Pylori infection and gastrointestinal complaints in a large cohort of symptomatic children". Acta Paediatr.

8- FaridF,RedaS,ELTehewyM et al, "The pattern of nephrotic s among Egyptian children"(1997).The Egyptian J.pediatri:14(1,2):71

9- MiyajiH , AzumaT , ItoS etal "H.Pylori infection occurs via close contact with infected individuals in early childhood"(2000):J Gastroenterol Hepatol , 15(3) : 257-62

10- Jones NL, Koletzko S, Goodman K, et al. Joint ESPGHAN/NASPGHAN

Guidelines for the management of pylori in children and adolescents (update 2016) . J Pediatr Gastroenterol Nutr 2017 ;64(6): 991-1003

11-SatoM, ShimoyamaT, TakahashiR, KajiyamaH, SanoY, SakaedaniN, KatoA, HirataH, FukudaY. Characterization and usefulness of stool antigen tests using a monoclonal antibody to Helicobacter pylori catalase. J Gastroenterol Hepatol. 2012;27 Suppl 3:23-28.

12- IbrahimM, SaidR, ELNabawyO and ShenoudaS “ Helicobacter Pylori Infection in Egyptian children with Nephrotic S" 2015, Nephrology Dialysis Transplantation, 30(3), Piii679.

13- Sabbagh P, Javanian M ,Koppolu V and etal "Helicobacter pylori infection in 
children":an overview of diagnostic methods : Eur J Clin ,Microbiol infect Dis.(2019) ; [Epub ahead of print ]
14- Moubri M , Buruca C Kalach N et al, “ performances of the IDEIA HpStAR stool Antigien Test in Detection of Helicobacter pylori infection before and after Eradication treatment in children" (2019):J trop pediatr .65(3) :210-216 